\title{
CREDIT RISK CONTRIBUTIONS UNDER THE VASICEK ONE-FACTOR MODEL: A FAST WAVELET EXPANSION APPROXIMATION
}

\author{
LUIS ORTIZ-GRACIA AND JOSEP J. MASDEMONT
}

\begin{abstract}
To measure the contribution of individual transactions inside the total risk of a credit portfolio is a major issue in financial institutions. VaR Contributions (VaRC) and Expected Shortfall Contributions (ESC) have become two popular ways of quantifying the risks. However, the usual Monte Carlo (MC) approach is known to be a very time consuming method for computing these risk contributions. In this paper we consider the Wavelet Approximation (WA) method for Value at Risk (VaR) computation presented in [Mas10] in order to calculate the Expected Shortfall (ES) and the risk contributions under the Vasicek one-factor model framework. We decompose the VaR and the ES as a sum of sensitivities representing the marginal impact on the total portfolio risk. Moreover, we present technical improvements in the Wavelet Approximation (WA) that considerably reduce the computational effort in the approximation while, at the same time, the accuracy increases.
\end{abstract}

\section{INTRODUCTION}

Credit risk concerns the risk of loss arising from an obliglor's inability to honor its obligations. Among other sources of risk, it is the most important one that a bank has to deal with due to big exposures concentrated in the portfolios. Because of this, financial institutions have to quantify credit risk at portfolio level.

We consider the Vasicek one-factor credit loss model as the default model which serves also as the basis of the Basel II (Basel Committee on Bank Supervision) internal rating based (IRB) approach. Under this model, defaults are driven by a latent common factor (business cycle or state of the economy) assumed to follow a standard normal distribution. It is also a one-period model, i.e., loss only occurs when an obligor defaults in a fixed time horizon.

The most common risk measures are Value-at-Risk and Expected Shortfall. As it is well known, VaR is not a coherent risk measure in the sense that is not sub-additive, in contradiction with the idea of diversification. In contrast, ES satisfies the four properties of a coherent risk measure (see [Art99] for a definition of these axioms). Both VaR and ES can be decomposed as a sum of sensitivities (see [Tas00]). These sensitivities, which are commonly named risk contributions, can be understood as the marginal impact on the risk of the total portfolio and are very important for loan pricing or asset allocation, to cite two examples.

In practice, each risk contribution is usually computed by means of $\mathrm{MC}$ calculated as the expected value of the loss distribution conditioned on a rare event, the VaR value, which represents an extreme loss for the credit portfolio. The usual Plain Monte Carlo presents practical inconveniences due to the large number of simulations required to get the rare events. Although in this context of MC simulation, [Gla05] develops efficient methods based on importance sampling to calculate VaR and ES contributions in the Vasicek multi-factor model, the computational effort is still very important. For this reason, analytical or fast numerical techniques are always welcome. One of such analytical techniques for VaR and VaRC computations is the saddle point (SP) method pioneered by Martin et al ([Mar01a] and [Mar01b]). They apply the approximation to the unconditional Moment Generating Function (MGF) and obtain accurate results at very small tail probabilities. This method is known to perform well with big portfolios at high loss levels. [Hua07a] compute the risk measures and 
contributions implementing a higher order saddle point method in the Vasicek model and apply the approximation to the conditional Moment Generating Function (MGF) instead of the unconditional MGF, where the saddle point works better, with an extra computational time. [Hua07b] present a comparative study for the calculation of VaR and VaRC with the SP method, MC with importance sampling (IS) and the normal approximation (NA) method. They conclude that there is not a perfect method that prevails among the others and the choice is a trade-off between speed, accuracy and robustness. NA is an accurate method and the fastest one but is not capable of handling with exposure concentration. IS is the most robust method but is highly demanding from a computational point of view when estimating the VaRC. The SP method preserves a good balance between speed and accuracy and is better than normal approximation to deal with exposure concentration. However, if the loss distribution is not smooth due to exceptional exposure concentration, a straightforward implementation of SP may be insufficient, and an adaptive SP should be employed instead. Alternatively, [Tak08] addresses the problem of calculating the marginal contributions using a numerical Laplace transform inversion of the MGF in the multi-sector setting and provide precise results in big size portfolios.

In this paper we extend the work undertaken with the estimation of the VaR value with the WA method in [Mas10] and we develop a new methodology for the computation of the ES, the VaR contributions and the ES contributions. We recall that this methodology approximate the credit loss cumulative distribution function (CDF) by a finite combination of Haar wavelets basis functions in order to invert the Laplace transform of the unconditional MFG. It was tested under the Vasicek one-factor model, showing accurate and fast results for a wide range of portfolios at very high loss levels. We will show that WA can get very accurate results even in presence of extremely exposure concentration when computing the risk measures and contributions, where a straightforward implementation of SP would fail. The key point for the calculation of the VaR, ES, VaRC and ESC is how to evaluate the coefficients of the wavelet expansion and their derivatives with respect to exposures. In the technical context, choosing a convenient path of integration in the Cauchy's integral formula, we can achieve more accurate results than [Mas10] at higher confidence levels without the need of doubling the number of subintervals when applying the trapezoidal rule for their calculation. Moreover, by means of truncating the integration variable in the Gauss-Hermite quadrature representing the business cycle, we get the same accuracy with fewer nodes, obtaining also a proportional reduction in the computational time. We point out that although we apply the WA method at scale ten in order to maintain accurate results in all the sample portfolios, the WA performs very well with smaller scales with a considerable reduction in the computational time (essentially is divided by two when moving from scale $m$ to $m-1$ ) .

The reminder of the paper is organized as follows. First we present the Vasicek model taken as framework in section two. In section three we do a short review of the Haar wavelets approach for the credit portfolio loss distribution. Section four is devoted to develop the new methodology for the ES and risk contributions. In section five an improved version of the GH formula is used to accelerate the algorithms, while numerical examples are shown in section six. Finally section seven is left to conclusions.

\section{The Model Framework}

To represent the uncertainty about future events, we specify a probability space $(\Omega, \mathcal{F}, \mathbb{P})$ with sample space $\Omega, \sigma$-algebra $\mathcal{F}$, probability measure $\mathbb{P}$ and with filtration $\left(\mathcal{F}_{t}\right)_{t \geq 0}$ satisfying the usual conditions. We fix a time horizon $T>0$. Usually $T$ equals one year.

Consider a credit portfolio consisting of $N$ obligors where each obligor $n$ is characterized by three parameters that we assume can be estimated by means of empirical default data: 
the exposure at default $E_{n}$, the loss given default, which without loss of generality we assume to be $100 \%$, and the probability of default $P_{n}$. The exposure at default of an obligor denotes the portion of exposure of the obligor that is lost in case of default. Let $D_{n}$ be the default indicator of obligor $n$ taking the following values.

$$
D_{n}= \begin{cases}1, & \text { if obligor } n \text { is in default, } \\ 0, & \text { if obligor } n \text { is not in default. }\end{cases}
$$

Let $\mathcal{L}$ be the portfolio loss given by:

$$
\mathcal{L}=\sum_{n=1}^{N} L_{n},
$$

where $L_{n}=E_{n} \cdot D_{n}$.

To test our methodology we consider the Vasicek one-factor Gaussian copula model as framework. The Vasicek model is a one period default model, i.e., loss only occurs when an obligor defaults in a fixed time horizon. Based on Merton's firm-value model, to describe the obligor's default and its correlation structure, we assign each obligor a random variable called firm-value. The firm-value of obligor $n$ is represented by a common, standard normally distributed factor $Y$ component and an idiosyncratic standard normal noise component $\epsilon_{n}$. The $Y$ factor is the state of the world or business cycle, usually called systematic factor.

$$
V_{n}(T)=\sqrt{\rho_{n}} Y+\sqrt{1-\rho_{n}} \epsilon_{n},
$$

where $Y$ and $\epsilon_{n}, \forall n \leq N$ are i.i.d. standard normally distributed.

In case that $\rho_{n}=\rho$ for all $n$, the parameter $\rho$ is called the common asset correlation. An important point is that, conditional on the realization of the systematic factor $Y$, the firm's values and defaults are independent. From now on, we assume $\rho_{n}$ to be constant.

Let us explain in detail the meaning of systematic and idiosyncratic risk. The first one can be seen as the macro-economic conditions and affects the credit-worthiness of all obligors simultaneously. The second one represents conditions inherent to each obligor and this is why they are assumed to be independent of each other.

In the Merton model an obligor $n$ defaults when its firm-value falls below a threshold level $T_{n}$ defined by $T_{n} \equiv \Phi^{-1}\left(P_{n}\right)$ where $\Phi(x)$ is the standard normal cumulative distribution function and $\Phi^{-1}(x)$ denotes its inverse. The probability of default of obligor $n$ conditional to a realization of $Y=y$ is given by

$$
p_{n}(y) \equiv \mathbb{P}\left(V_{n}<T_{n} \mid Y=y\right)=\Phi\left(\frac{T_{n}-\sqrt{\rho} y}{\sqrt{1-\rho}}\right) .
$$

Consequently, the conditional probability of default depends on the systematic factor, reflecting the fact that the business cycle affects the possibility of an obligor's default.

\section{HaAr Wavelets Approach}

Let $F$ be the cumulative distribution function of $\mathcal{L}$. Without loss of generality, we can assume $\sum_{n=1}^{N} E_{n}=1$ and for a given $E=\left(E_{1}, \ldots, E_{N}\right)$ let us consider,

$$
F(E, x)= \begin{cases}\bar{F}(E, x), & \text { if } 0 \leqslant x \leqslant 1 \\ 1, & \text { if } x>1\end{cases}
$$


for a certain $\bar{F}$ defined in $[0,1]$. Following the work in [Mas10], we approximate the distribution function by a finite sum of Haar wavelets basis functions with convergence in $L^{2}([0,1])$,

$$
\bar{F}(E, x) \simeq \bar{F}_{m}(E, x), \quad \bar{F}_{m}(E, x)=\sum_{k=0}^{2^{m}-1} c_{m, k}(E) \phi_{m, k}(x),
$$

where $m$ is the scale of the approximation and $k$ is the translation parameter.

The unconditional moment generating function,

$$
M(E, s) \equiv \mathbb{E}\left(e^{-s \mathcal{L}}\right)=\mathbb{E}\left(\mathbb{E}\left(e^{-s \mathcal{L}} \mid Y\right)\right)=\int_{\mathbb{R}} \prod_{n=1}^{N}\left[1-p_{n}(y)+p_{n}(y) e^{-s E_{n}}\right] \frac{1}{\sqrt{2 \pi}} e^{-\frac{y^{2}}{2}} d y
$$

is also the Laplace transform of the density function $f$ of $\mathcal{L}$,

$$
M(E, s) \equiv \mathbb{E}\left(e^{-s \mathcal{L}}\right)=\int_{0}^{+\infty} e^{-s x} f(E, x) d x .
$$

Integrating by parts the expression (3) and using the approximation in (1), the coefficients $c_{m, k}$ are recovered by the Laplace transform inversion, giving us the expressions,

$$
c_{m, 0}=\frac{\int_{\mathbb{R}} \prod_{n=1}^{N}\left[1-p_{n}(y)\right] \frac{1}{\sqrt{2 \pi}} e^{-\frac{y^{2}}{2}} d y}{2^{\frac{m}{2}}}
$$

and

$$
c_{m, k}(E)=\frac{2}{\pi r^{k}} \int_{0}^{\pi} \Re\left(Q\left(E, r e^{i u}\right)\right) \cos (k u) d u, \quad k=1, \ldots, 2^{m}-1
$$

where

$$
Q(E, z) \equiv \sum_{k=0}^{2^{m}-1} c_{m, k}(E) z^{k} \simeq \frac{M\left(E,-2^{m} \ln (z)\right)-z^{2^{m}}}{2^{\frac{m}{2}}(1-z)} .
$$

In [Mas10] the coefficients $c_{m, k}$ are accurately computed by means of the ordinary trapezoidal rule and the MGF is evaluated using Gauss-Hermite formulae with 20 nodes. The results show a fast convergence towards the value obtained by means of a Monte Carlo method with five million sampling scenarios, which serves as a benchmark. However, the speed of the algorithm is highly conditioned by the number of times that we have to evaluate the MGF, i.e., the number of subintervals in the trapezoidal rule, the number of nodes used in Gauss-Hermite integration and the size of the portfolio. It has been proved that $2^{m}$ subintervals are enough to integrate (4) using the trapezoidal rule, getting accurate VaR values at $99.9 \%$ and $99.99 \%$ confidence levels. However, for higher loss levels it is necessary to consider more subintervals to get acceptable relative errors, and consequently this also increases the computational time. In section 5 we present an improved version of the algorithm used in [Mas10] which again reduces significantly the computational cost when a large number of nodes of GH are required, or when the portfolio contains a big number of obligors. Moreover more accurate results are found at very high confidence levels without increasing the number of subintervals in the trapezoidal rule.

\section{Credit Risk Measures and Contributions}

In this section we recall the calculation of the VaR value and present a numerical formula for the estimation of the Expected Shortfall using the wavelet approximation method of the previous section. We also apply this method to compute the marginal contribution of the obligors to the total risk at portfolio level. 
4.1. VaR and Expected Shortfall. Let us consider a portfolio with exposures $E=$ $\left(E_{1}, \ldots, E_{N}\right)$ and let $\alpha \in(0,1)$ be a given confidence level. The $\alpha$-quantile of the loss distribution of $\mathcal{L}$ in this context, is called Value at Risk. This is,

$$
\operatorname{VaR}(\mathrm{E})_{\alpha}=\inf \{l \in \mathbb{R}: \mathbb{P}(\mathcal{L} \leq l) \geq \alpha\}=\inf \{l \in \mathbb{R}: F(E, l) \geq \alpha\},
$$

where the $\alpha$ of interest are usually very close to 1 . This is the measure chosen in the Basel II Accord for the computation of capital requirement, meaning that a bank, managing its risks according to Basel II, must reserve capital by an amount of $\operatorname{VaR}(\mathrm{E})_{\alpha}$ to cover extreme losses.

Considering a wavelet approximation in a level of resolution $m$, the VaR value at confidence level $\alpha$ calculated by the WA method is of the form $\operatorname{VaR}(\mathrm{E})_{\alpha}^{W(m)}=\frac{2 \bar{k}+1}{2^{m+1}}$ for a certain $\bar{k} \in\left\{0,1, \ldots, 2^{m}-1\right\}$, where $\bar{k}$ is such that $\bar{F}_{m}\left(E, \operatorname{VaR}(\mathrm{E})_{\alpha}^{W(m)}\right) \simeq \alpha$, (see [Mas10] for a detailed description of the method).

A crucial property for a coherent risk measure is the sub-additivity condition. As mentioned previously, the VaR measure fails to satisfy this condition although the measure is widely used in practice. This can be explained by the fact that when distributions are normal, or close to normal, it can be shown that VaR and ES are quite close and behave similarly. However, as soon as a distribution is characterized by a long tail behavior, the similarity between VaR and ES does not hold anymore. In this case, employing the VaR measure may lead to a considerable underestimation of risk.

By definition, the Expected Shortfall at confidence level $\alpha$ is given by,

$$
\mathrm{ES}(\mathrm{E})_{\alpha}=\frac{1}{1-\alpha} \int_{\operatorname{VaR}(E)_{\alpha}}^{+\infty} x f(E, x) d x .
$$

Then, integrating by parts and using the approximation in (1) we have,

$$
\mathrm{ES}(\mathrm{E})_{\alpha}=\frac{1}{1-\alpha}\left(1-\alpha \operatorname{VaR}(\mathrm{E})_{\alpha}-\int_{V a R(E)_{\alpha}}^{1} \bar{F}(E, x) d x\right) \simeq \mathrm{ES}(\mathrm{E})_{\alpha}^{W(m)},
$$

where

$$
\mathrm{ES}(\mathrm{E})_{\alpha}^{W(m)} \equiv \frac{1}{1-\alpha}\left(1-\alpha \operatorname{VaR}(\mathrm{E})_{\alpha}^{W(m)}-\frac{1}{2^{\frac{m}{2}+1}} c_{m, \bar{k}}(E)-\frac{1}{2^{\frac{m}{2}}} \sum_{k=\bar{k}+1}^{2^{m}-1} c_{m, k}(E)\right)
$$

For sake of clarity, from now on the explicit dependence of all ES and VaR expressions that appear in next sections with respect to $E$ will be dropped.

4.2. VaR Contributions and Expected Shortfall Contributions. Let us consider how to decompose the total risk into individual transactions. We carry out the allocation principle given by the partial derivative of the risk measure with respect to the exposure of an obligor. In this way we define the risk contribution of obligor $i$ to the VaR value at confidence level $\alpha$ by,

$$
\operatorname{VaRC}_{\alpha, i} \equiv E_{i} \cdot \frac{\partial \mathrm{VaR}_{\alpha}}{\partial E_{i}}
$$

which satisfies the additivity condition, $\sum_{i=1}^{N} \mathrm{VaRC}_{\alpha, i}=\mathrm{VaR}_{\alpha}$ (see [Tas00] for details).

Taking into account that $\bar{F}\left(E, \operatorname{VaR}_{\alpha}\right)=\alpha$ then,

$$
\operatorname{VaRC}_{\alpha, i} \equiv E_{i} \cdot \frac{\partial \mathrm{VaR}_{\alpha}}{\partial E_{i}}=-E_{i} \cdot \frac{\frac{\partial \bar{F}\left(E, \mathrm{VaR}_{\alpha}\right)}{\partial E_{i}}}{\left.\frac{\bar{F}(E, x)}{\partial x}\right|_{x=\mathrm{VaR}_{\alpha}}}=-E_{i} \cdot \frac{\frac{\partial \bar{F}\left(E, \mathrm{VaR}_{\alpha}\right)}{\partial E_{i}}}{f\left(E, \mathrm{VaR}_{\alpha}\right)}
$$


Now according to $(1), \bar{F}(E, x) \simeq \sum_{k=0}^{2^{m}-1} c_{m, k}(E) \phi_{m, k}(x)$. Taking partial derivatives with respect $E_{i}$ we get,

$$
\frac{\partial \bar{F}(E, x)}{\partial E_{i}} \simeq \sum_{k=0}^{2^{m}-1} \frac{\partial c_{m, k}(E)}{\partial E_{i}} \phi_{m, k}(x)
$$

and evaluating this expression in $\mathrm{VaR}_{\alpha}$ and using the approximation to the $\mathrm{VaR}$ value given by $\operatorname{VaR}_{\alpha}^{W(m)}$, we have,

$$
\frac{\partial \bar{F}\left(E, \mathrm{VaR}_{\alpha}\right)}{\partial E_{i}} \simeq \sum_{k=0}^{2^{m}-1} \frac{\partial c_{m, k}(E)}{\partial E_{i}} \phi_{m, k}\left(\operatorname{VaR}_{\alpha}\right) \simeq \sum_{k=0}^{2^{m}-1} \frac{\partial c_{m, k}(E)}{\partial E_{i}} \phi_{m, k}\left(\operatorname{VaR}_{\alpha}^{W(m)}\right)=2^{\frac{m}{2}} \frac{\partial c_{m, \bar{k}}(E)}{\partial E_{i}}
$$

due to the fact that

$$
\phi_{m, k}(x)= \begin{cases}2^{\frac{m}{2}} & \text { if } \frac{k}{2^{m}} \leq x<\frac{k+1}{2^{m}} \\ 0 & \text { elsewhere }\end{cases}
$$

Finally, considering the expressions (7) and (9) we obtain,

$$
\operatorname{VaRC}_{\alpha, i} \simeq \operatorname{VaRC}_{\alpha, i}^{W(m)}
$$

where $\operatorname{VaRC}_{\alpha, i}^{W(m)} \equiv \mathcal{C} \cdot E_{i} \cdot \frac{\partial c_{m, \bar{k}}(E)}{\partial E_{i}}$ and $\mathcal{C}$ is a constant such that $\sum_{i=1}^{N} \operatorname{VaRC}_{\alpha, i}^{W(m)}=\operatorname{VaR}_{\alpha}^{W(m)}$.

In a similar way we define the ESC for the obligor $i$ at confidence level $\alpha$ as,

$$
\mathrm{ESC}_{\alpha, i} \equiv E_{i} \cdot \frac{\partial \mathrm{ES}_{\alpha}}{\partial E_{i}}
$$

satisfying the additivity condition $\sum_{i=1}^{N} \mathrm{ESC}_{\alpha, i}=\mathrm{ES}_{\alpha}$. For the computation of the expected shortfall contributions we have taken the derivative of the expected shortfall expression in (6) with respect to $E_{i}$ and used the approximation (8). That is,

$$
\begin{aligned}
\mathrm{ESC}_{\alpha, i} & \equiv E_{i} \cdot \frac{\partial \mathrm{ES}_{\alpha}}{\partial E_{i}}=E_{i} \cdot \frac{1}{1-\alpha}\left(-\alpha \frac{\partial \mathrm{VaR}_{\alpha}}{\partial E_{i}}+\frac{\partial \operatorname{VaR}_{\alpha}}{\partial E_{i}} \bar{F}\left(E, \operatorname{VaR}_{\alpha}\right)-\int_{\operatorname{VaR}_{\alpha}}^{1} \frac{\partial \bar{F}(E, x)}{\partial E_{i}} d x\right) \\
& \simeq \mathrm{ESC}_{\alpha, i}^{W(m)},
\end{aligned}
$$

where

$$
\mathrm{ESC}_{\alpha, i}^{W(m)} \equiv-E_{i} \cdot \frac{1}{2^{\frac{m}{2}}} \cdot \frac{1}{1-\alpha} \cdot\left(\frac{1}{2} \frac{\partial c_{m, \bar{k}}(E)}{\partial E_{i}}+\sum_{k=\bar{k}+1}^{2^{m}-1} \frac{\partial c_{m, k}(E)}{\partial E_{i}}\right)
$$

Later, in the numerical examples section, we will test the accuracy of the WA method for the calculation of VARC and ESC by means of Monte Carlo estimates, which will serve us as a benchmark. Under appropriate conditions the marginal VaR contribution at confidence level $\alpha$ of the obligor $i$ is

$$
\operatorname{VaRC}_{\alpha, i}=\mathbb{E}\left(L_{i} \mid \mathcal{L}=\operatorname{VaR}_{\alpha}\right)
$$

and the marginal contribution at confidence level $\alpha$ to the expected shortfall is,

$$
\mathrm{ESC}_{\alpha, i}=\mathbb{E}\left(L_{i} \mid \mathcal{L} \geq \operatorname{VaR}_{\alpha}\right) .
$$

Thus, in both cases, the marginal risk contributions are conditional expectations of the individual loss random variables, conditioned on rare values of the portfolio loss $\mathcal{L}$. Moreover, it can be shown that,

$$
\sum_{i=1}^{N} \mathbb{E}\left(L_{i} \mid \mathcal{L}=\operatorname{VaR}_{\alpha}\right)=\operatorname{VaR}_{\alpha} \quad \text { and } \quad \sum_{i=1}^{N} \mathbb{E}\left(L_{i} \mid \mathcal{L} \geq \operatorname{VaR}_{\alpha}\right)=\mathrm{ES}_{\alpha}
$$


We estimate risk contributions by means of Monte Carlo simulations in two steps. First, we compute the VaR value through an ordinary Monte Carlo simulation and then we use the estimated VaR in (12) and (13). In this second step, for a given loss level $l$, we consider the problem of estimating $\mathbb{E}\left(L_{i} \mid \mathcal{L}=l\right)$ and $\mathbb{E}\left(L_{i} \mid \mathcal{L} \geq l\right)$. Both cases can be treated together considering $C_{i}=\mathbb{E}\left(L_{i} \mid \mathcal{L} \in \mathcal{A}\right)$ where $\mathcal{A}=l$ or $\mathcal{A}=[l, \infty)$. For each sampled scenario we proceed as follows,

(1) Generate the systemic factor $Y$.

(2) For each obligor generate the idiosyncratic component $\epsilon_{i}, i=1, \ldots, N$.

(3) Finally, generate the default indicators $D_{i}$ and the individual losses $L_{i}, \quad i=1, \ldots, N$. These steps are repeated till $K$ independent scenarios are generated. Let $L^{(k)}=L_{1}^{(k)}+\ldots+L_{N}^{(k)}$ be the total portfolio loss on the $k$-th replication. To estimate the risk contributions $C_{i}$, we use,

$$
\hat{C}_{i}=\frac{\sum_{k=1}^{K} L_{i}^{(k)} \chi_{\left\{L^{(k)} \in \mathcal{A}\right\}}}{\sum_{k=1}^{K} \chi_{\left\{L^{(k)} \in \mathcal{A}\right\}}} .
$$

To measure the variability of this estimator we use the following proposition (since $\hat{C}_{i}$ is a ratio estimator, we can not use a simple standard deviation to measure its precision. For a detailed explanation of the MC method for risk contributions see [Gla05]).

Proposition 1. Suppose $\mathbb{P}(L \in \mathcal{A})>0$ and let

$$
\hat{\sigma}_{i}^{2}=\frac{K \sum_{k=1}^{K}\left(L_{i}^{(k)}-\hat{C}_{i}\right)^{2} \chi_{\left\{L^{(k)} \in \mathcal{A}\right\}}}{\left(\sum_{k=1}^{K} \chi_{\left\{L^{(k)} \in \mathcal{A}\right\}}\right)^{2}},
$$

taking the ratio to be zero whenever the denominator is zero. Then the distribution of $\frac{\hat{C}_{i}-C_{i}}{\hat{\sigma}_{i} / \sqrt{K}}$ converges to the standard normal and $\left(\hat{C}_{i}-z_{\delta / 2} \hat{\sigma}_{i} / \sqrt{K}, \hat{C}_{i}+z_{\delta / 2} \hat{\sigma}_{i} / \sqrt{K}\right)$ is an asymptotically valid $1-\delta$ confidence interval for $C_{i}$, with $\Phi\left(z_{\delta / 2}\right)=1-\delta / 2$.

\section{An Improved Gauss-Hermite Integration Formula}

An important issue regarding the computation of the coefficients $c_{m, k}$ in (4) and $\frac{\partial c_{m, k}\left(E_{i}\right)}{\partial E_{i}}$ in (8) is the way to compute $\Re\left(Q\left(E, r e^{i u}\right)\right)$ and $\frac{\partial \Re\left(Q\left(E, r e^{i u}\right)\right)}{\partial E_{i}}$ for a fixed loss level $u$. In this section we present, in addition, a simplification in the GH formula that considerable reduces the computational effort.

5.1. Fast computation of $c_{m, k}(E)$. Let us explain in detail the computation of the coefficients in the WA method. First we take the expression (5),

$$
Q\left(E, r e^{i u}\right)=\frac{M\left(E,-2^{m} \ln \left(r e^{i u}\right)\right)-\left(r e^{i u}\right)^{2^{m}}}{2^{m / 2}\left(1-r e^{i u}\right)},
$$

where $M$ is the unconditional Moment Generating Function.

If we define $z_{1}=M\left(E,-2^{m} \ln \left(r e^{i u}\right)\right)-\left(r e^{i u}\right)^{2^{m}}$ and $z_{2}=2^{m / 2}\left(1-r e^{i u}\right)$ then we have,

$$
\Re\left(Q\left(E, r e^{i u}\right)\right)=\frac{\Re\left(z_{1}\right) \Re\left(z_{2}\right)+\Im\left(z_{1}\right) \Im\left(z_{2}\right)}{\left(\Re\left(z_{2}\right)\right)^{2}+\left(\Im\left(z_{2}\right)\right)^{2}},
$$

where

$$
\Re\left(z_{1}\right)=\Re\left(M\left(E,-2^{m} \ln \left(r e^{i u}\right)\right)\right)-r^{2^{m}} \cos \left(2^{m} u\right), \quad \Re\left(z_{2}\right)=2^{m / 2}(1-r \cos (u)),
$$

and

$$
\Im\left(z_{1}\right)=\Im\left(M\left(E,-2^{m} \ln \left(r e^{i u}\right)\right)\right)-r^{2^{m}} \sin \left(2^{m} u\right), \quad \Im\left(z_{2}\right)=-2^{m / 2} r \sin (u) .
$$


Proceeding this way we have to find an expression for $\Re\left(M\left(E,-2^{m} \ln \left(r e^{i u}\right)\right)\right)$ and $\Im\left(M\left(E,-2^{m} \ln \left(r e^{i u}\right)\right)\right)$. Introducing the change of variable $y=\sqrt{2} x$ in (2) we obtain,

$$
M(E, s)=\int_{\mathbb{R}} \prod_{n=1}^{N}\left[1-p_{n}(\sqrt{2} x)+p_{n}(\sqrt{2} x) e^{-s E_{n}}\right] \frac{1}{\sqrt{\pi}} e^{-x^{2}} d x .
$$

We can approximate this integral by means of a Gauss-Hermite formula,

$$
M(E, s) \simeq \sum_{j=1}^{l / 2} a_{j}\left(\bar{M}\left(E, s ; x_{j}^{-}\right)+\bar{M}\left(E, s ; x_{j}^{+}\right)\right)
$$

where $\bar{M}(E, s ; x)=\frac{1}{\sqrt{\pi}} \prod_{n=1}^{N}\left[1-p_{n}(\sqrt{2} x)+p_{n}(\sqrt{2} x) e^{-s E_{n}}\right]$ and $x_{j}^{-}=-x_{j}^{+}$and $a_{j}$ are respectively the nodes and weights of the quadrature. This is, we can compute,

$\Re\left(M\left(E,-2^{m} \ln \left(r e^{i u}\right)\right)\right) \simeq \sum_{j=1}^{l / 2} a_{j}\left(\Re\left(\bar{M}\left(E,-2^{m} \ln \left(r e^{i u}\right) ; x_{j}^{-}\right)\right)+\Re\left(\bar{M}\left(E,-2^{m} \ln \left(r e^{i u}\right) ; x_{j}^{+}\right)\right)\right)$,

and

$\Im\left(M\left(E,-2^{m} \ln \left(r e^{i u}\right)\right)\right) \simeq \sum_{j=1}^{l / 2} a_{j}\left(\Im\left(\bar{M}\left(E,-2^{m} \ln \left(r e^{i u}\right) ; x_{j}^{-}\right)\right)+\Im\left(\bar{M}\left(E,-2^{m} \ln \left(r e^{i u}\right) ; x_{j}^{+}\right)\right)\right)$.

We notice that,

$\bar{M}\left(E,-2^{m} \ln \left(r e^{i u}\right) ; x\right)=\frac{1}{\sqrt{\pi}} \prod_{n=1}^{N}\left[1-p_{n}(\sqrt{2} x)+p_{n}(\sqrt{2} x) r^{2^{m} E_{n}}\left(\cos \left(2^{m} E_{n} u\right)+i \sin \left(2^{m} E_{n} u\right)\right)\right]$.

So using polar coordinates this expression casts into,

$$
\bar{M}\left(E,-2^{m} \ln \left(r e^{i u}\right) ; x\right)=\frac{1}{\sqrt{\pi}} \prod_{n=1}^{N}\left(R_{n}\right)_{\theta_{n}}=\frac{1}{\sqrt{\pi}}\left(\prod_{n=1}^{N} R_{n}\right)_{\sum_{n=1}^{N} \theta_{n}}
$$

where $R_{n}=\left|z_{n}\right|, \theta_{n}=\arctan \left(\frac{\Im\left(z_{n}\right)}{\Re\left(z_{n}\right)}\right)$ and

$$
z_{n}=1-p_{n}(\sqrt{2} x)+p_{n}(\sqrt{2} x) r^{2^{m} E_{n}}\left(\cos \left(2^{m} E_{n} u\right)+i \sin \left(2^{m} E_{n} u\right)\right) .
$$

Finally, expanding these expressions we conclude,

$$
\begin{gathered}
R_{n}=\sqrt{\left(1-p_{n}(\sqrt{2} x)\right)^{2}+r^{2^{m+1} E_{n}} p_{n}^{2}(\sqrt{2} x)+2 p_{n}(\sqrt{2} x)\left(1-p_{n}(\sqrt{2} x)\right) r^{2^{m} E_{n}} \cos \left(2^{m} E_{n} u\right)} \\
\theta_{n}=\arctan \left(\frac{r^{2^{m} E_{n}} p_{n}(\sqrt{2} x) \sin \left(2^{m} E_{n} u\right)}{1-p_{n}(\sqrt{2} x)+p_{n}(\sqrt{2} x) r^{2^{m} E_{n}} \cos \left(2^{m} E_{n} u\right)}\right)
\end{gathered}
$$

$$
\begin{aligned}
& \Re\left(\bar{M}\left(E,-2^{m} \ln \left(r e^{i u}\right) ; x\right)\right)=\frac{1}{\sqrt{\pi}}\left(\prod_{n=1}^{N} R_{n}\right) \cos \left(\sum_{n=1}^{N} \theta_{n}\right), \\
& \Im\left(\bar{M}\left(E,-2^{m} \ln \left(r e^{i u}\right) ; x\right)\right)=\frac{1}{\sqrt{\pi}}\left(\prod_{n=1}^{N} R_{n}\right) \sin \left(\sum_{n=1}^{N} \theta_{n}\right) .
\end{aligned}
$$


There are three key points that essentially determine the computational complexity of the Wavelet Approximation method. The first one is the portfolio size, $N$, which is fixed. The second one is the number of times that the MGF must be evaluated $\left(2^{m}+1\right)$ and the last one the number of nodes $(l)$ to be used in the GH quadrature every time the MGF is computed at a loss level $u$. Let us show in the following paragraphs an interesting fact regarding this last point.

We remark that, in practice, financial companies tend to calibrate the parameter of probability of default of a company from their rating systems and they get in this way several pools. This means that portfolio obligors are classified in rating categories, being the parameter $P_{n}$ identical for all the obligors inside the same group, and distinct among different groups.

For sake of simplicity, and without loss of generality, let us assume that we have a unique rating category over the whole portfolio. This is $P_{n}=P$ for all $n=1, \ldots, N$, and consequently, $p_{n}(y)=p(y)$ for all $n=1, \ldots, N$. We observe that under this hypothesis,

$$
\lim _{p \rightarrow 0} R_{n}=1, \quad \quad \lim _{p \rightarrow 0} \theta_{n}=0,
$$

and

$$
\lim _{p \rightarrow 1} R_{n}=r^{2^{m} E_{n}}, \quad \quad \lim _{p \rightarrow 1} \theta_{n}=2^{m} E_{n} u .
$$

Then, given a tolerance $\epsilon$,

$$
\Re\left(\bar{M}\left(E,-2^{m} \ln \left(r e^{i u}\right) ; x\right)\right) \simeq \frac{1}{\sqrt{\pi}}, \quad \Im\left(\bar{M}\left(E,-2^{m} \ln \left(r e^{i u}\right) ; x\right)\right) \simeq 0,
$$

for all $x \geq x_{1}$ where $x_{1}$ is such that $p\left(\sqrt{2} x_{1}\right)<\epsilon$. This is, when $y>\frac{\Phi^{-1}(P)-\sqrt{1-\rho} \Phi^{-1}(\epsilon)}{\sqrt{\rho}}$.

In a similar way,

$\Re\left(\bar{M}\left(E,-2^{m} \ln \left(r e^{i u}\right) ; x\right)\right) \simeq \frac{1}{\sqrt{\pi}} r^{2^{m}} \cos \left(2^{m} u\right), \quad \Im\left(\bar{M}\left(E,-2^{m} \ln \left(r e^{i u}\right) ; x\right)\right) \simeq \frac{1}{\sqrt{\pi}} r^{2^{m}} \sin \left(2^{m} u\right)$, for all $x \leq x_{2}$ where $x_{2}$ is such that $p\left(\sqrt{2} x_{2}\right)>1-\epsilon$, i.e. when $y<\frac{\Phi^{-1}(P)-\sqrt{1-\rho} \Phi^{-1}(1-\epsilon)}{\sqrt{\rho}}$.

Taking into account these facts we have that (15) and (16) can be computed as,

$$
\begin{aligned}
& \Re\left(M\left(E,-2^{m} \ln \left(r e^{i u}\right)\right)\right) \simeq \\
& \simeq \sum_{j=1}^{l / 2-n_{2}} a_{j} \Re\left(\bar{M}\left(E,-2^{m} \ln \left(r e^{i u}\right) ; x_{j}^{-}\right)\right)+\sum_{j=1}^{l / 2-n_{1}} a_{j} \Re\left(\bar{M}\left(E,-2^{m} \ln \left(r e^{i u}\right) ; x_{j}^{+}\right)\right)+ \\
& \quad+\frac{1}{\sqrt{\pi}}\left(n_{1}+n_{2} r^{2^{m}} \cos \left(2^{m} u\right)\right),
\end{aligned}
$$

and

$$
\begin{aligned}
& \Im\left(M\left(E,-2^{m} \ln \left(r e^{i u}\right)\right)\right) \simeq \\
& \simeq \sum_{j=1}^{l / 2-n_{2}} a_{j} \Im\left(\bar{M}\left(E,-2^{m} \ln \left(r e^{i u}\right) ; x_{j}^{-}\right)\right)+\sum_{j=1}^{l / 2-n_{1}} a_{j} \Im\left(\bar{M}\left(E,-2^{m} \ln \left(r e^{i u}\right) ; x_{j}^{+}\right)\right)+ \\
& \quad+\frac{1}{\sqrt{\pi}} n_{2} r^{2^{m}} \sin \left(2^{m} u\right),
\end{aligned}
$$

where $n_{1}$ (respectively $n_{2}$ ) is the number of nodes in the Gauss-Hermite quadrature greater (respectively smaller) or equal than $x_{1}$ (respectively $x_{2}$ ). Thus, given a tolerance $\epsilon$ we have truncated the left and right tails of the integration variable representing the business cycle making use of the limit behavior instead of continuing with the quadrature. In the section devoted to numerical examples we show the large amount of computation time saved this way, while the accuracy remains the same. 
5.2. Fast computation of $\frac{\partial c_{m, k}(E)}{\partial E_{n}}$. Let us explain the computations of the partial derivatives of the coefficients of the WA with respect to the exposures. Again in this case we perform a truncation of the integration variable in a similar way as in the previous section.

Taking the derivative of (4) with respect to $E_{n}$ we get,

$$
\frac{\partial c_{m, k}(E)}{\partial E_{n}}=\frac{2}{\pi r^{k}} \int_{0}^{\pi} \frac{\partial \Re\left(Q\left(E, r e^{i u}\right)\right)}{\partial E_{n}} \cos (k u) d u .
$$

Then taking into account (14) we have,

$$
\frac{\partial \Re\left(Q\left(E, r e^{i u}\right)\right)}{\partial E_{n}}=\frac{\frac{\partial \Re\left(M\left(E,-2^{m} \ln \left(r e^{i u}\right)\right)\right)}{\partial E_{n}} \Re\left(z_{2}\right)+\frac{\partial \Im\left(M\left(E,-2^{m} \ln \left(r e^{i u}\right)\right)\right)}{\partial E_{n}} \Im\left(z_{2}\right)}{\left(\Re\left(z_{2}\right)\right)^{2}+\left(\Im\left(z_{2}\right)\right)^{2}},
$$

and again using the Gauss-Hermite quadrature we have,

$$
\begin{aligned}
& \frac{\partial \Re\left(M\left(E,-2^{m} \ln \left(r e^{i u}\right)\right)\right)}{\partial E_{n}} \simeq \\
& \sum_{j=1}^{l / 2} a_{j}\left(\frac{\partial \Re\left(\bar{M}\left(E,-2^{m} \ln \left(r e^{i u}\right) ; x_{j}^{-}\right)\right)}{\partial E_{n}}+\frac{\partial \Re\left(\bar{M}\left(E,-2^{m} \ln \left(r e^{i u}\right) ; x_{j}^{+}\right)\right)}{\partial E_{n}}\right),
\end{aligned}
$$

and

$$
\begin{aligned}
& \frac{\partial \Im\left(M\left(E,-2^{m} \ln \left(r e^{i u}\right)\right)\right)}{\partial E_{n}} \simeq \\
& \sum_{j=1}^{l / 2} a_{j}\left(\frac{\partial \Im\left(\bar{M}\left(E,-2^{m} \ln \left(r e^{i u}\right) ; x_{j}^{-}\right)\right)}{\partial E_{n}}+\frac{\partial \Im\left(\bar{M}\left(E,-2^{m} \ln \left(r e^{i u}\right) ; x_{j}^{+}\right)\right)}{\partial E_{n}}\right) .
\end{aligned}
$$

Now following steps similar to the former section one obtains,

$$
\begin{aligned}
& \frac{\partial \Re\left(\bar{M}\left(E,-2^{m} \ln \left(r e^{i u}\right) ; x\right)\right)}{\partial E_{n}}=\frac{1}{\sqrt{\pi}}\left(\prod_{\underline{n}=1}^{N} R_{\underline{n}}\right)\left[\frac{1}{R_{n}} \frac{\partial R_{n}}{\partial E_{n}} \cos \left(\sum_{\underline{n}=1}^{N} \theta_{\underline{n}}\right)-\frac{\partial \theta_{n}}{\partial E_{n}} \sin \left(\sum_{\underline{n}=1}^{N} \theta_{\underline{n}}\right)\right], \\
& \frac{\partial \Im\left(\bar{M}\left(E,-2^{m} \ln \left(r e^{i u}\right) ; x\right)\right)}{\partial E_{n}}=\frac{1}{\sqrt{\pi}}\left(\prod_{\underline{n}=1}^{N} R_{\underline{n}}\right)\left[\frac{1}{R_{n}} \frac{\partial R_{n}}{\partial E_{n}} \sin \left(\sum_{\underline{n}=1}^{N} \theta_{\underline{n}}\right)+\frac{\partial \theta_{n}}{\partial E_{n}} \cos \left(\sum_{\underline{n}=1}^{N} \theta_{\underline{n}}\right)\right], \\
& \frac{\partial R_{n}}{\partial E_{n}}= \\
& \frac{2^{m} r^{2^{m} E_{n}}}{R_{n}}\left(r^{2^{m} E_{n}} p_{n}^{2}(\sqrt{2} x) \ln r+p_{n}(\sqrt{2} x)\left(1-p_{n}(\sqrt{2} x)\right)\left(\ln r \cos \left(2^{m} E_{n} u\right)-u \sin \left(2^{m} E_{n} u\right)\right)\right), \\
& \frac{\partial \theta_{n}}{\partial E_{n}}= \\
& \frac{2^{m} r^{2^{m} E_{n}} p_{n}(\sqrt{2} x)\left(\theta_{n}^{D}\left(\ln r \sin \left(2^{m} E_{n} u\right)+\cos \left(2^{m} E_{n} u\right) u\right)-\theta_{n}^{N}\left(\ln r \cos \left(2^{m} E_{n} u\right)-u \sin \left(2^{m} E_{n} u\right)\right)\right)}{\left(\theta_{n}^{N}\right)^{2}+\left(\theta_{n}^{D}\right)^{2}},
\end{aligned}
$$

with,

$$
\begin{gathered}
\theta_{n}^{N}=r^{2^{m} E_{n}} p_{n}(\sqrt{2} x) \sin \left(2^{m} E_{n} u\right), \\
\theta_{n}^{D}=1-p_{n}(\sqrt{2} x)+p_{n}(\sqrt{2} x) r^{2^{m} E_{n}} \cos \left(2^{m} E_{n} u\right) .
\end{gathered}
$$


Again we try to reduce the computational effort in the Gauss-Hermite quadratures (21) and (22). To this end we take into account that,

$$
\lim _{p \rightarrow 0} \frac{\partial R_{n}}{\partial E_{n}}=0, \quad \quad \quad \lim _{p \rightarrow 0} \frac{\partial \theta_{n}}{\partial E_{n}}=0
$$

and

$$
\lim _{p \rightarrow 1} \frac{\partial R_{n}}{\partial E_{n}}=2^{m} r^{2^{m} E_{n}} \ln r, \quad \lim _{p \rightarrow 1} \frac{\partial \theta_{n}}{\partial E_{n}}=2^{m} u .
$$

Then, given a tolerance $\bar{\epsilon}$ we have,

$$
\frac{\partial \Re\left(\bar{M}\left(E,-2^{m} \ln \left(r e^{i u}\right) ; x\right)\right)}{\partial E_{n}} \simeq 0, \quad \frac{\partial \Im\left(\bar{M}\left(E,-2^{m} \ln \left(r e^{i u}\right) ; x\right)\right)}{\partial E_{n}} \simeq 0,
$$

for all $x \geq \bar{x}_{1}$ where $\bar{x}_{1}$ is such that $p\left(\sqrt{2} \bar{x}_{1}\right)<\bar{\epsilon}$, i.e. $y>\frac{\Phi^{-1}(P)-\sqrt{1-\rho} \Phi^{-1}(\bar{\epsilon})}{\sqrt{\rho}}$.

Also,

$$
\begin{aligned}
\frac{\partial \Re\left(\bar{M}\left(E,-2^{m} \ln \left(r e^{i u}\right) ; x\right)\right)}{\partial E_{n}} & \simeq \frac{1}{\sqrt{\pi}} 2^{m} r^{2^{m}}\left(\ln r \cos \left(2^{m} u\right)-u \sin \left(2^{m} u\right)\right), \\
\frac{\partial \Im\left(\bar{M}\left(E,-2^{m} \ln \left(r e^{i u}\right) ; x\right)\right)}{\partial E_{n}} & \simeq \frac{1}{\sqrt{\pi}} 2^{m} r^{2^{m}}\left(\ln r \sin \left(2^{m} u\right)+u \cos \left(2^{m} u\right)\right),
\end{aligned}
$$

for all $x \leq \bar{x}_{2}$ where $\bar{x}_{2}$ is such that $p\left(\sqrt{2} \bar{x}_{2}\right)<\bar{\epsilon}$, i.e. $y<\frac{\Phi^{-1}(P)-\sqrt{1-\rho} \Phi^{-1}(\bar{\epsilon})}{\sqrt{\rho}}$.

Taking into account these facts, (21) and (22) can be computed by means of,

$$
\begin{aligned}
& \frac{\partial \Re\left(M\left(E,-2^{m} \ln \left(r e^{i u}\right)\right)\right)}{\partial E_{n}} \simeq \\
& \simeq \sum_{j=1}^{l / 2-\bar{n}_{2}} a_{j} \frac{\partial \Re\left(\bar{M}\left(E,-2^{m} \ln \left(r e^{i u}\right) ; x_{j}^{-}\right)\right)}{\partial E_{n}}+\sum_{j=1}^{l / 2-\bar{n}_{1}} a_{j} \frac{\partial \Re\left(\bar{M}\left(E,-2^{m} \ln \left(r e^{i u}\right) ; x_{j}^{+}\right)\right)}{\partial E_{n}} \\
& \quad+\frac{1}{\sqrt{\pi}} \bar{n}_{2} 2^{m} r^{2^{m}}\left(\ln r \cos \left(2^{m} u\right)-u \sin \left(2^{m} u\right)\right),
\end{aligned}
$$

and

$$
\begin{aligned}
& \frac{\partial \Im\left(M\left(E,-2^{m} \ln \left(r e^{i u}\right)\right)\right)}{\partial E_{n}} \simeq \\
& \simeq \sum_{j=1}^{l / 2-\bar{n}_{2}} a_{j} \frac{\partial \Im\left(\bar{M}\left(E,-2^{m} \ln \left(r e^{i u}\right) ; x_{j}^{-}\right)\right)}{\partial E_{n}}+\sum_{j=1}^{l / 2-\bar{n}_{1}} a_{j} \frac{\partial \Im\left(\bar{M}\left(E,-2^{m} \ln \left(r e^{i u}\right) ; x_{j}^{+}\right)\right)}{\partial E_{n}} \\
& \quad+\frac{1}{\sqrt{\pi}} \bar{n}_{2} 2^{m} r^{2^{m}}\left(\ln r \sin \left(2^{m} u\right)+u \cos \left(2^{m} u\right)\right),
\end{aligned}
$$

where $\bar{n}_{1}$ (respectively $\bar{n}_{2}$ ) is the number of nodes in the Gauss-Hermite quadrature greater (respectively smaller) or equal than $\bar{x}_{1}$ (respectively $\bar{x}_{2}$ ). Again, given a tolerance $\bar{\epsilon}$, we have truncated the left and right tails of the quadrature in the variable of the business cycle and used the limit behavior instead.

This method is specially suitable for very big portfolios or when the GH quadrature would require a considerable number of nodes. The improvements will be shown in the next section devoted to numerical examples. 


\section{Numerical EXAMPLES}

We test ${ }^{1}$ the methodology for the computation of ES, VaRC and ESC developed in the previous sections considering four different portfolios. All them with exposure concentrations and ranging from 10 to 10000 obligors. The common set of parameters used for the Wavelet Approximation method are: scale $m=10,2^{m}$ intervals for the trapezoidal quadrature in the coefficients formula (4) and $r=0.9995$.

Portfolio 1. This portfolio has $N=10000$ obligors with $\rho=0.15, P_{n}=0.01$ and $E_{n}=\frac{1}{n}$ for $n=1, \ldots, N$, as in [Mas10].

Portfolio 2. This portfolio has $N=1001$ obligors, with $E_{n}=1$ for $n=1, \ldots, 1000$, and one obligor with $E_{1001}=100 . P_{n}=0.0033$ for all the obligors and $\rho=0.2$ as in [Hua07b].

Portfolio 3. This portfolio has $N=100$ obligors, all them with $P_{n}=0.01, \rho=0.5$ and exposures,

$$
E_{n}= \begin{cases}1, & n=1, \ldots, 20 \\ 4, & n=21, \ldots, 40 \\ 9, & n=41, \ldots, 60 \\ 16, & n=61, \ldots, 80 \\ 25, & n=81, \ldots, 100\end{cases}
$$

as in [Gla05].

\begin{tabular}{lccll}
\hline \hline Method & $l / 2-n_{2}$ & $l / 2-n_{1}$ & $\alpha=0.9999$ & $\alpha=0.99999$ \\
\hline \hline $\operatorname{VaR}_{\alpha}^{M}$ & & & 0.2267 & 0.2973 \\
$\operatorname{VaR}_{\alpha}^{A}$ & & & $0.1683(-25.76 \%)$ & $0.2322(-21.91 \%)$ \\
$\operatorname{VaR}_{\alpha}^{W(10)}(20)([$ Mas10]) & 10 & 10 & $0.2280(0.60 \%)$ & $0.3306(11.18 \%)$ \\
$\operatorname{VaR}_{\alpha}^{W(10)}(20)$ & 10 & 10 & $0.2261(-0.26 \%)$ & $0.2935(-1.30 \%)$ \\
$\operatorname{VaR}_{\alpha}^{W(10)}\left(20,4 \cdot 10^{-1}\right)$ & 9 & 0 & $0.2261(-0.26 \%)$ & $0.2944(-0.97 \%)$ \\
$\operatorname{VaR}_{\alpha}^{W(10)}\left(20,6 \cdot 10^{-1}\right)$ & 7 & 0 & $0.2261(-0.26 \%)$ & $0.2944(-0.97 \%)$ \\
\hline
\end{tabular}

TABLE 1. VaR values at $99.99 \%$ and $99.999 \%$ confidence levels for portfolio 1. Errors relative to Monte Carlo are shown in parenthesis.

Portfolio 4. This portfolio has $N=10$ obligors, all them with $\rho=0.5, P_{n}=0.0021$ and $E_{n}=\frac{1}{n}$ for $n=1, \ldots, N$ as in [Mas10].

For practical purposes and without loss of generality, in all cases we normalize dividing $E_{n}$ by $\sum_{n=1}^{N} E_{n}$ to meet the condition $\sum_{n=1}^{N} E_{n}=1$.

From now on, let $\operatorname{VaR}_{\alpha}^{M}, \mathrm{ES}_{\alpha}^{M}$ be the VaR and $\mathrm{ES}$ values computed by means of a Plain Monte Carlo simulation with 5 million scenarios and $\operatorname{VaRC}_{\alpha, i}^{M}, \mathrm{ESC}_{\alpha, i}^{M}$ the $\mathrm{VaRC}$ and $\mathrm{ESC}$ values computed by means of a Plain Monte Carlo simulation with 100 million scenarios to be used as a benchmark.

In what follows let us denote by $\operatorname{VaR}_{\alpha}^{W(m)}(l), \operatorname{ES}_{\alpha}^{W(m)}(l), \operatorname{VaRC}_{\alpha, i}^{W(m)}(l), \operatorname{ESC}_{\alpha, i}^{W(m)}(l)$ the result of the Wavelet Approximation method for computing risk measures and contributions,

\footnotetext{
${ }^{1}$ Computations have been carried out sequentially in a personal computer Dell Vostro 320 under GNU/Linux OS, Intel CPU Core 2 E7500, 2.93GHz, 4GB RAM and using the gcc compiler with optimization level 2.
} 


\begin{tabular}{lcclll}
\hline \hline Method & $l / 2-n_{2}$ & $l / 2-n_{1}$ & $\alpha=0.99$ & $\alpha=0.999$ & $\alpha=0.9999$ \\
\hline \hline $\operatorname{ES}_{\alpha}^{M}$ & & & 0.1290 & 0.1895 & 0.2553 \\
$\operatorname{ES}_{\alpha}^{W(10)}(20)$ & 10 & 10 & $0.1290(-0.02 \%)$ & $0.1895(-0.01 \%)$ & $0.2556(0.12 \%)$ \\
$\operatorname{ES}_{\alpha}^{W(10)}\left(20,4 \cdot 10^{-1}\right)$ & 9 & 0 & $0.1289(-0.12 \%)$ & $0.1895(-0.01 \%)$ & $0.2556(0.12 \%)$ \\
$\operatorname{ES}_{\alpha}^{W(10)}\left(20,6 \cdot 10^{-1}\right)$ & 7 & 0 & $0.1289(-0.11 \%)$ & $0.1896(0.00 \%)$ & $0.2559(0.25 \%)$ \\
\hline
\end{tabular}

TABLE 2. ES values at 99\%, 99.9\% and 99.99\% confidence levels for portfolio 1 . Errors relative to Monte Carlo are shown in parenthesis.

remarking that, we use a Gauss-Hermite quadrature with $l$ nodes for the integrals (15) and (16) in the case of VaR and ES, and also for the integrals (21) and (22) in the case of VaRC and ESC. Analogously, $\operatorname{VaR}_{\alpha}^{W(m)}(l, \epsilon), \operatorname{ES}_{\alpha}^{W(m)}(l, \epsilon), \operatorname{VaRC}_{\alpha, i}^{W(m)}(l, \bar{\epsilon}), \operatorname{ESC}_{\alpha, i}^{W(m)}(l, \bar{\epsilon})$ denote Wavelet Approximation results when computing risk measures and risk contributions considering $l$-nodes in the Gauss-Hermite quadrature, but using the expressions (19) and (20) in the case of VaR and ES, and (23), (24) in the case of VaRC and ESC.

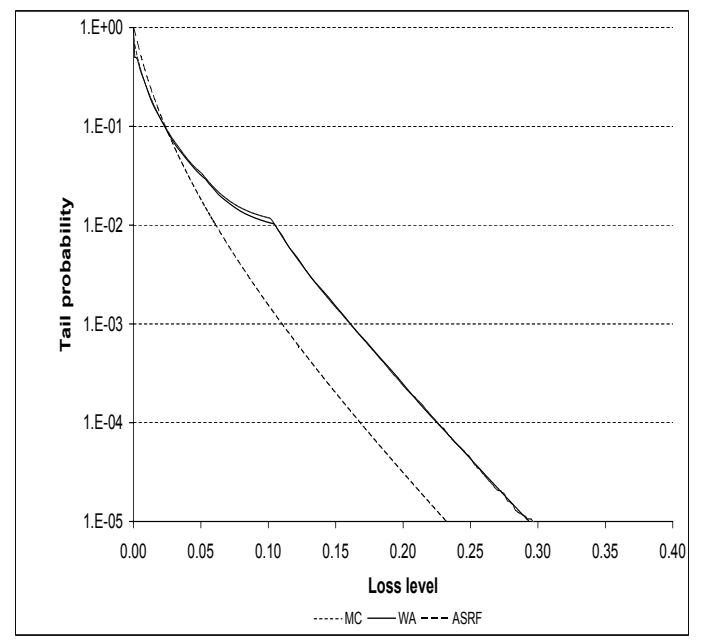

FiguRE 1. Tail probability approximation of portfolio 1 with WA using a 20nodes GH formula and $\epsilon=6 \cdot 10^{-1}$.

Let $\operatorname{VaR}_{\alpha}^{A}, \mathrm{ES}_{\alpha}^{A}, \operatorname{VaRC}_{\alpha, i}^{A}, \mathrm{ESC}_{\alpha, i}^{A}$ be the risk measures and contributions evaluated by the Asymptotic Single Risk Factor (ASRF) model (further details about this method can be found in [Lut09]). Table 1 presents the very high confidence VaR values for portfolio 1 computed by means of Monte Carlo, ASRF and the Wavelet Approximation method. For comparison we provide the results obtained with WA reported in [Mas10] (and denoted by $\operatorname{VaR}_{\alpha}^{W(10)}(20)$ $\left(\left[\right.\right.$ Mas10])) which differs from $\operatorname{VaR}_{\alpha}^{W(10)}(20)$ in the choice of the parameter $r$. The number of negative $\left(l / 2-n_{2}\right)$ and positive $\left(l / 2-n_{1}\right)$ nodes where the conditional MGF is evaluated are also specified. The relative error presented for the VaR value at $99.999 \%$ confidence level is about $-1 \%$ when using the WA method with $r=0.9995$ in contrast with the error reported in [Mas10] which is $11.88 \%$. Figure 1 represents the loss distribution for the WA method with a 20 -nodes GH formula and $\epsilon=6 \cdot 10^{-1}$, which only requires the evaluation of the 

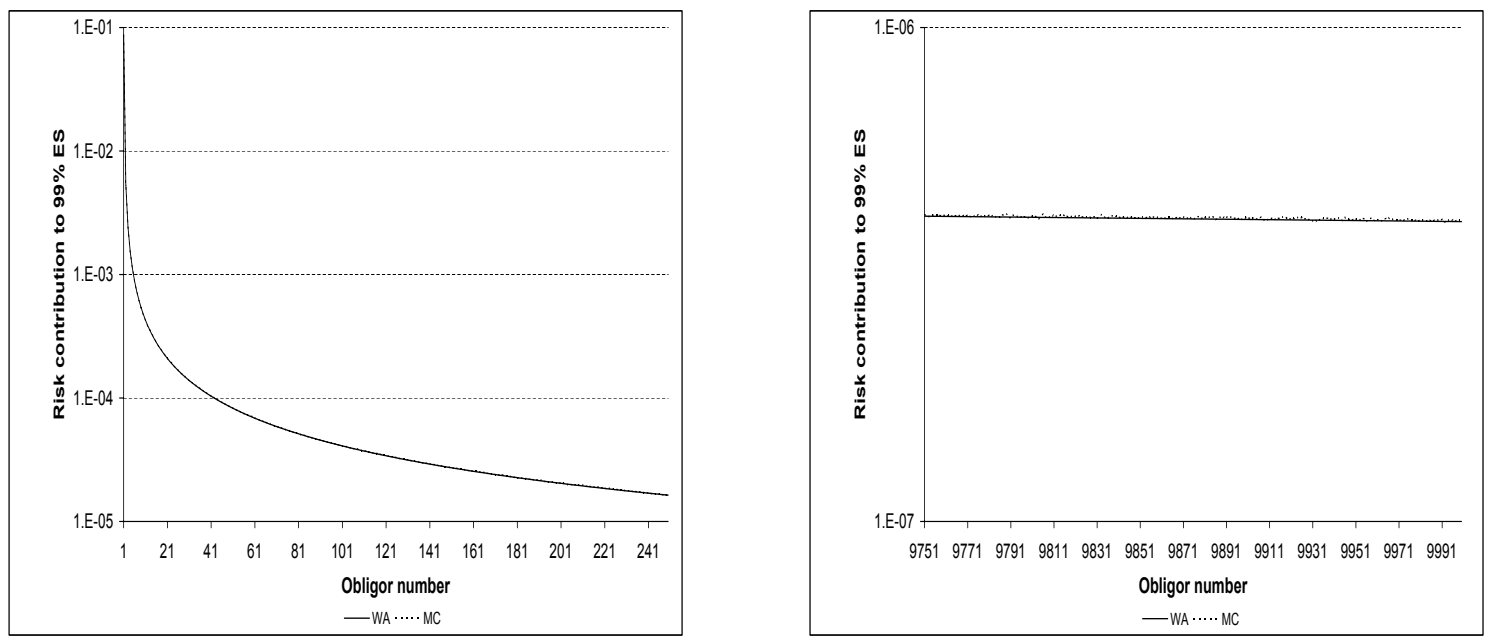

FiguRE 2. Risk contributions to the ES at $99 \%$ confidence level for the portfolio 1.

MGF in the first 7 negative nodes to achieve a high precision. We also provide the result for the ASRF method and Monte Carlo (which, as always, give us as the benchmark). The estimation of $\mathrm{VaR}$ by means of the $\operatorname{VaR}_{\alpha}^{W(10)}\left(20,6 \cdot 10^{-1}\right)$ approximation requires 25.3 seconds of CPU time, while the $\operatorname{VaR}_{\alpha}^{W(10)}(20)$ and $\operatorname{VaR}_{\alpha}^{W(10)}(20)$ ([Mas10]) approximations need of 71.5 seconds. This is, the implementation of the asymptotic truncation of sections 5.1 and 5.2 represents an important improvement. It is also worth to underline that $\operatorname{VaR}_{0.9999}^{W(9)}\left(20,6 \cdot 10^{-1}\right)$ and $\operatorname{VaR}_{0.9999}^{W(8)}\left(20,6 \cdot 10^{-1}\right)$ give also very accurate results (with relative errors equal to $-0.47 \%$ and $-0.90 \%$ respectively) and computation times of 12.7 and 6.4 seconds respectively. We also want to remark that the ASRF method clearly underestimates the risk due to the presence of name concentration.
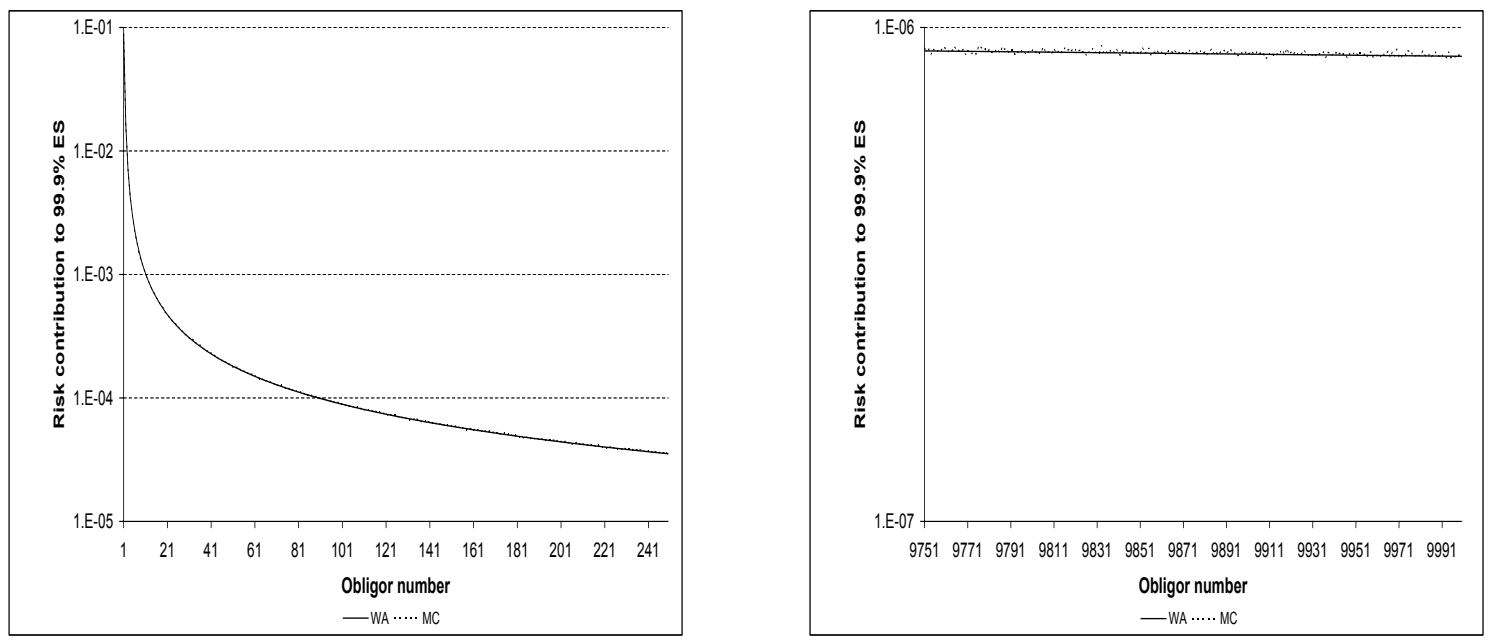

Figure 3. Risk contributions to the ES at $99.9 \%$ confidence level for portfolio 1. 
The expected shortfall calculated at several confidence levels with $\operatorname{ES}_{\alpha}^{W(10)}\left(20,6 \cdot 10^{-1}\right)$ is presented in table 2 (we omit the computational time because there is almost no difference between VaR and ES calculation in terms of computational effort). As the results show again, a high precision is achieved in terms of the relative error. Figures 2 and 3 represent the contributions to the expected shortfall at $99 \%$ and $99.9 \%$ confidence levels respectively by means of the WA method with a 20-nodes $\mathrm{GH}$ formula and $\bar{\epsilon}=10^{-4}$ (we present the ESC instead of the VaRC due to the robustness of plain Monte Carlo simulation for the first measure). For sake of clarity in the plots, we have only represented the 250 biggest and smallest risk contributions. The convergence towards Monte Carlo is clear and the sum of the risk contributions shown in table 3 are very close to the ES values given in table 2 . $\mathrm{ESC}_{\alpha, n}^{W(10)}\left(20,1 \cdot 10^{-4}\right)$ takes 622.5 seconds of CPU to evaluate the partial derivative of the MGF in 14 nodes, while the $\operatorname{ESC}_{\alpha, n}^{W(10)}(20)$ method needs of 671.8 seconds.

\begin{tabular}{lcccl}
\hline \hline Method & $l / 2-\bar{n}_{2}$ & $l / 2-\bar{n}_{1}$ & $\alpha=0.99$ & $\alpha=0.999$ \\
\hline \hline$\sum_{n=1}^{N} \operatorname{ESC}_{\alpha, n}^{M}$ & & & 0.1290 & 0.1892 \\
$\sum_{n=1}^{N} \operatorname{ESC}_{\alpha, n}^{W(10)}(20)$ & 10 & 10 & 0.1293 & 0.1891 \\
$\sum_{n=1}^{N} \operatorname{ESC}_{\alpha, n}^{W(10)}\left(20,1 \cdot 10^{-4}\right)$ & 10 & 4 & 0.1293 & 0.1890 \\
\hline
\end{tabular}

TABLE 3. Comparison of the total ES contributions at $99 \%$ and $99.9 \%$ confidence levels for portfolio 1 .

Next we consider portfolio 2 which is a well diversified portfolio where a big exposure (representing about $9 \%$ of the total portfolio exposure) has been added. So it presents exposure concentration. As pointed out in [Hua07b], a straightforward saddle point approximation fails for all the quantiles preceding the point of non smoothness. However, WA method is capable to deal with this problem as we illustrate in figure 4.

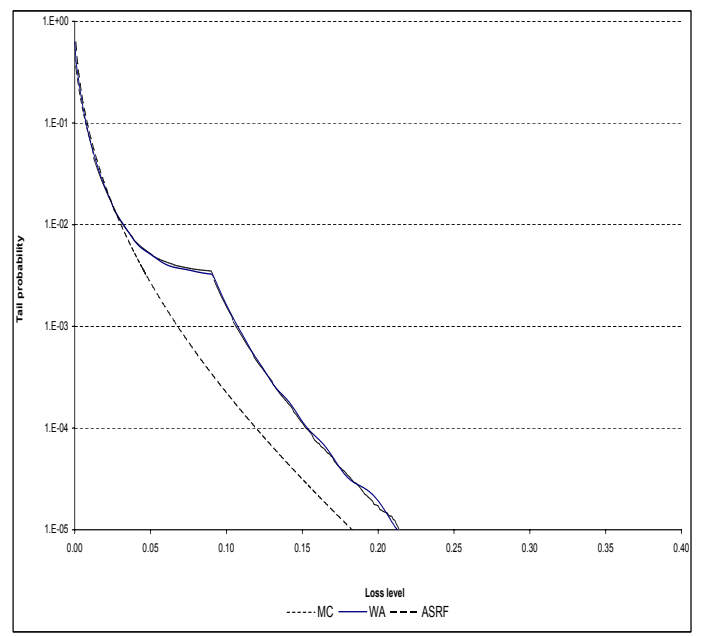

FigURE 4. Tail probability approximation for portfolio 2 with WA using a 64-nodes GH quadrature and $\epsilon=5 \cdot 10^{-1}$.

We also note that we obtain the same accuracy with the evaluation of only 15 nodes of the asymptotic formulae (which needs only 5.6 seconds of CPU time) and using the full one with 64 nodes (which needs of 23.4 seconds). The risk contributions to the $99.9 \% \mathrm{ES}$ are 


\begin{tabular}{lccll}
\hline \hline Method & $l / 2-n_{2}$ & $l / 2-n_{1}$ & $\alpha=0.999$ & $\alpha=0.9999$ \\
\hline \hline $\operatorname{VaR}_{\alpha}^{M}$ & & & 0.1077 & 0.1532 \\
$\operatorname{VaR}_{\alpha}^{A}$ & & & $0.0679(-37.00 \%)$ & $0.1195(-21.99 \%)$ \\
$\operatorname{VaR}_{\alpha}^{W(10)}(64)$ & 32 & 32 & $0.1079(0.18 \%)$ & $0.1538(0.41 \%)$ \\
$\operatorname{VaR}_{\alpha}^{W(10)}\left(64,10^{-8}\right)$ & 32 & 13 & $0.1079(0.18 \%)$ & $0.1538(0.41 \%)$ \\
$\operatorname{VaR}_{\alpha}^{W(10)}\left(64,10^{-4}\right)$ & 30 & 3 & $0.1079(0.18 \%)$ & $0.1538(0.41 \%)$ \\
$\operatorname{VaR}_{\alpha}^{W(10)}\left(64,10^{-2}\right)$ & 25 & 0 & $0.1079(0.18 \%)$ & $0.1538(0.41 \%)$ \\
$\operatorname{VaR}_{\alpha}^{W(10)}\left(64,5 \cdot 10^{-1}\right)$ & 15 & 0 & $0.1079(0.18 \%)$ & $0.1538(0.41 \%)$ \\
\hline $\operatorname{ES}_{\alpha}^{M}$ & & & 0.1274 & 0.1809 \\
$\operatorname{ES}_{\alpha}^{W(10)}(64)$ & 32 & 32 & $0.1273(-0.02 \%)$ & $0.1810(0.09 \%)$ \\
$\operatorname{ES}_{\alpha}^{W(10)}\left(64,10^{-8}\right)$ & 32 & 13 & $0.1273(-0.02 \%)$ & $0.1810(0.09 \%)$ \\
$\operatorname{ES}_{\alpha}^{W(10)}\left(64,10^{-4}\right)$ & 30 & 3 & $0.1273(-0.02 \%)$ & $0.1810(0.09 \%)$ \\
$\operatorname{ES}_{\alpha}^{W(10)}\left(64,10^{-2}\right)$ & 25 & 0 & $0.1273(-0.02 \%)$ & $0.1810(0.09 \%)$ \\
$\operatorname{ES}_{\alpha}^{W(10)}\left(64,5 \cdot 10^{-1}\right)$ & 15 & 0 & $0.1273(-0.02 \%)$ & $0.1810(0.09 \%)$ \\
\hline
\end{tabular}

TABLE 4. VaR and ES values at $99.9 \%$ and $99.99 \%$ confidence levels for portfolio 2. Errors relative to Monte Carlo are shown in parenthesis.

also provided in table 5 using different $\bar{\epsilon}$. We present only the biggest and the smallest risk contributions. Relative errors are almost identical with 33 and 64 nodes and the computation times are 78.4 and 103.5 seconds. It is important to mention that, in practice, we only need to compute the contributions for two different exposures. However, we have performed the calculations for the whole portfolio in order to have an idea of the computational effort for a portfolio of this size.

Let us consider now the portfolio 3 . The plot of figure 5 shows the portfolio loss distribution approximated with the WA method with a 64 -nodes GH formula and $\epsilon=10^{-1}$. Table 6 contains different scenarios changing the parameter $\epsilon$. It is remarkable the high precision achieved when using $\epsilon=10^{-1}$, which means that the conditional MGF has been just evaluated in 12 negative nodes. The $\operatorname{VaR}_{\alpha}^{W(10)}(64)$ approximation needs 2.3 seconds while the approximation $\operatorname{VaR}_{\alpha}^{W(10)}\left(64,10^{-1}\right)$ needs only 0.6 seconds. The $99.9 \%$ VaR contributions are presented in table 7 and plotted in figure 6. To compute the VaR contributions by means of Monte Carlo simulation we have considered $\mathcal{A}=\left(\mathrm{VaR}_{\alpha}^{M}-5 \cdot 10^{-4}, \mathrm{VaR}_{\alpha}^{M}+5 \cdot 10^{-4}\right)$ instead of $\mathcal{A}=\operatorname{VaR}_{\alpha}^{M}$, due to the fact that $\mathrm{VaR}$ is a rare event. Moreover, we have generated $99 \%$ confidence intervals for the risk contributions as detailed in proposition 1 . The risk contributions calculated by the WA method using a 64-nodes GH formula and $\bar{\epsilon}=10^{-4}$ lie in the $99 \%$ MC confidence intervals, showing again the excellent accuracy of the method. The ES contributions at $99.9 \%$ and $99.99 \%$ confidence levels are presented in tables 8 and 9 respectively (and plotted in figure 7). The $\operatorname{ESC}_{\alpha, n}^{W(10)}\left(64,10^{-4}\right)$ approximation shows very accurate results except for the 20 smallest exposures at confidence level $99.99 \%$ which considerably underestimates the risk. The $\operatorname{ESC}_{\alpha, n}^{W(10)}(64)$ method takes 8 seconds for the computations while $\mathrm{ESC}_{\alpha, n}^{W(10)}\left(64,10^{-4}\right)$ needs of 4.5 seconds. Like in the previous example, we have calculated all the risk contributions. 


\begin{tabular}{lccl}
\hline \hline Method & $l / 2-\bar{n}_{2}$ & $l / 2-\bar{n}_{1}$ & $\alpha=0.999$ \\
\hline \hline $\operatorname{ESC}_{\alpha, 1001}^{M}$ & 32 & 32 & 0.082016 \\
$\operatorname{ESC}_{\alpha, 1001}^{W(10)}(64)$ & 32 & 13 & $0.081075(-1.15 \%)$ \\
$\operatorname{ESC}_{\alpha, 1001}^{W(10)}\left(64,10^{-8}\right)$ & 32 & 9 & $0.081075(-1.15 \%)$ \\
$\operatorname{ESC}_{\alpha, 1001}^{W(10)}\left(64,10^{-6}\right)$ & 30 & 3 & $0.081075(-1.15 \%)$ \\
$\operatorname{ESC}_{\alpha, 1001}^{W(10)}\left(64,10^{-4}\right)$ & & & 0.000045 \\
\hline $\operatorname{ESC}_{\alpha, 1}^{M}$ & 32 & 32 & $0.000046(2.04 \%)$ \\
$\operatorname{ESC}_{\alpha, 1}^{W(10)}(64)$ & 32 & 32 & $0.000046(2.04 \%)$ \\
$\operatorname{ESC}_{\alpha, 1}^{W(10)}\left(64,10^{-8}\right)$ & 32 & 9 & $0.000046(2.04 \%)$ \\
$\operatorname{ESC}_{\alpha, 1}^{W(10)}\left(64,10^{-6}\right)$ & 30 & 3 & $0.000046(1.92 \%)$ \\
$\operatorname{ESC}_{\alpha, 1}^{W(10)}\left(64,10^{-4}\right)$ & & & 0.1274 \\
\hline$\sum_{n=1}^{N} \operatorname{ESC}_{\alpha, n}^{M}$ & 32 & 32 & $0.1274(-0.01 \%)$ \\
$\sum_{n=1}^{N} \operatorname{ESC}_{\alpha, n}^{W(10)}(64)$ & 32 & 32 & $0.1274(-0.01 \%)$ \\
$\sum_{n=1}^{N} \operatorname{ESC}_{\alpha, n}^{W(10)}\left(64,10^{-8}\right)$ & 32 & 9 & $0.1274(-0.01 \%)$ \\
$\sum_{n=1}^{N} \operatorname{ESC}_{\alpha, n}^{W(10)}\left(64,10^{-6}\right)$ & 30 & 3 & $0.1273(-0.05 \%)$ \\
$\sum_{n=1}^{N} \operatorname{ESC}_{\alpha, n}^{W(10)}\left(64,10^{-4}\right)$ & & &
\end{tabular}

TABLE 5. ES contributions at 99.9\% confidence level for portfolio 2. Errors relative to Monte Carlo are shown in parenthesis.

\begin{tabular}{lccll}
\hline \hline Method & $l / 2-n_{2}$ & $l / 2-n_{1}$ & $\alpha=0.999$ & $\alpha=0.9999$ \\
\hline \hline $\operatorname{VaR}_{\alpha}^{M}$ & & & 0.4350 & 0.6859 \\
$\operatorname{VaR}_{\alpha}^{A}$ & & & $0.4209(-3.25 \%)$ & $0.6661(-2.89 \%)$ \\
$\operatorname{VaR}_{\alpha}^{W(10)}(64)$ & 32 & 32 & $0.4341(-0.21 \%)$ & $0.6870(0.16 \%)$ \\
$\operatorname{VaR}_{\alpha}^{W(10)}\left(64,10^{-8}\right)$ & 22 & 6 & $0.4341(-0.21 \%)$ & $0.6870(0.16 \%)$ \\
$\operatorname{VaR}_{\alpha}^{W(10)}\left(64,10^{-4}\right)$ & 17 & 1 & $0.4341(-0.21 \%)$ & $0.6870(0.16 \%)$ \\
$\operatorname{VaR}_{\alpha}^{W(10)}\left(64,10^{-2}\right)$ & 14 & 0 & $0.4341(-0.21 \%)$ & $0.6870(0.16 \%)$ \\
$\operatorname{VaR}_{\alpha}^{W(10)}\left(64,10^{-1}\right)$ & 12 & 0 & $0.4341(-0.21 \%)$ & $0.6870(0.16 \%)$ \\
\hline $\operatorname{ES}_{\alpha}^{M}$ & & & 0.5445 & 0.7576 \\
$\operatorname{ES}_{\alpha}^{W(10)}(64)$ & 32 & 32 & $0.5449(0.08 \%)$ & $0.7621(0.59 \%)$ \\
$\operatorname{ES}_{\alpha}^{W(10)}\left(64,10^{-8}\right)$ & 22 & 6 & $0.5449(0.08 \%)$ & $0.7621(0.59 \%)$ \\
$\operatorname{ES}_{\alpha}^{W(10)}\left(64,10^{-4}\right)$ & 17 & 1 & $0.5449(0.08 \%)$ & $0.7621(0.59 \%)$ \\
$\operatorname{ES}_{\alpha}^{W(10)}\left(64,10^{-2}\right)$ & 14 & 0 & $0.5449(0.08 \%)$ & $0.7621(0.59 \%)$ \\
$\operatorname{ES}_{\alpha}^{W(10)}\left(64,10^{-1}\right)$ & 12 & 0 & $0.5450(0.09 \%)$ & $0.7624(0.63 \%)$ \\
\hline
\end{tabular}

TABLE 6. VaR and ES values at $99.9 \%$ and $99.99 \%$ confidence levels for portfolio 3. Errors relative to Monte Carlo are shown in parenthesis. 

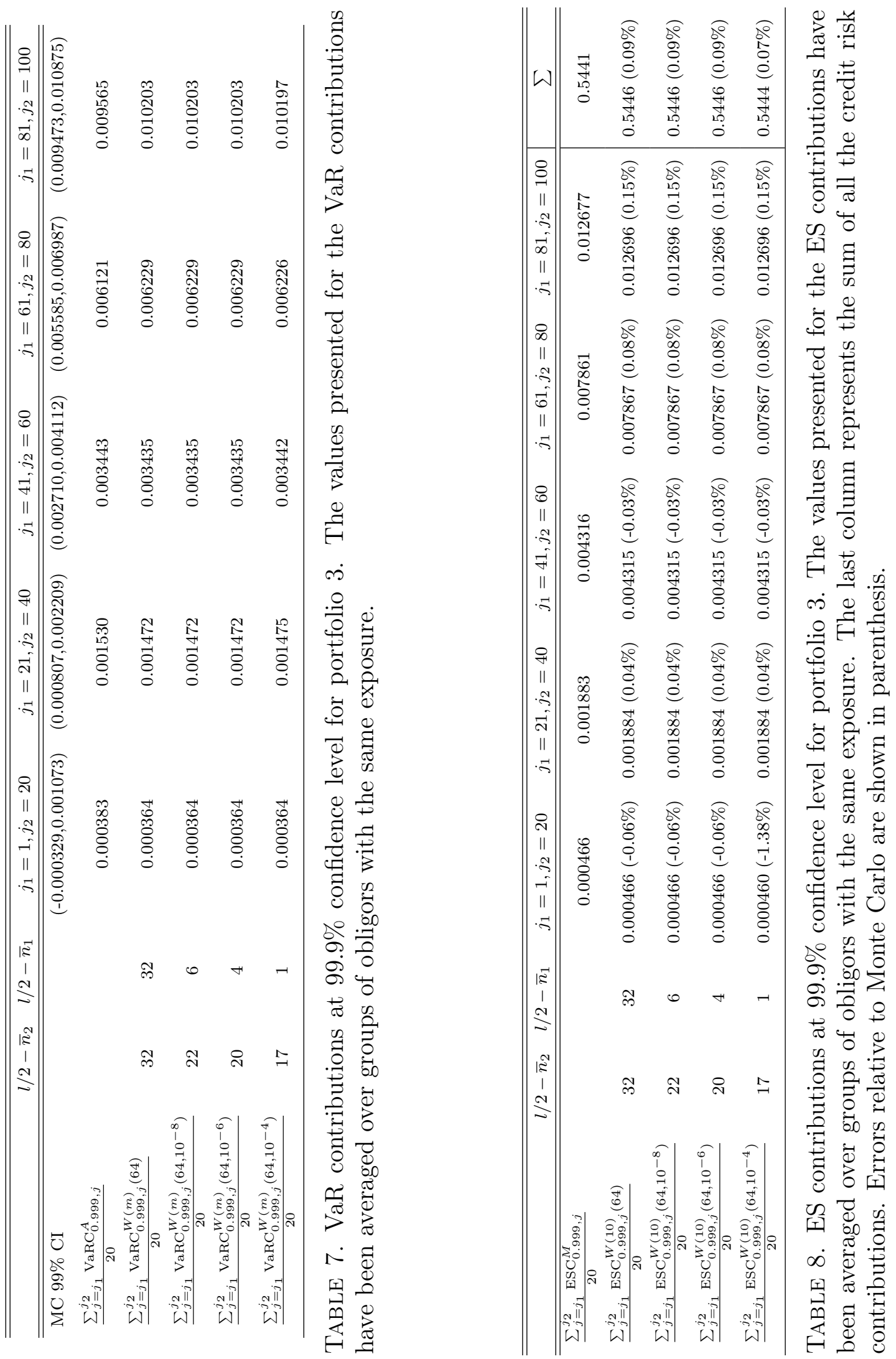


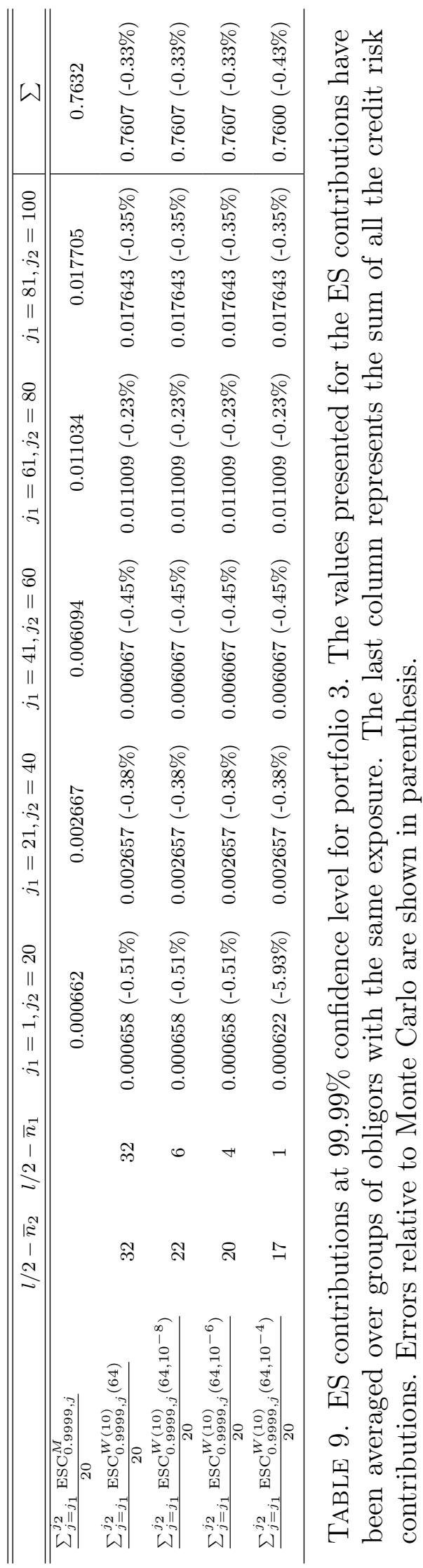




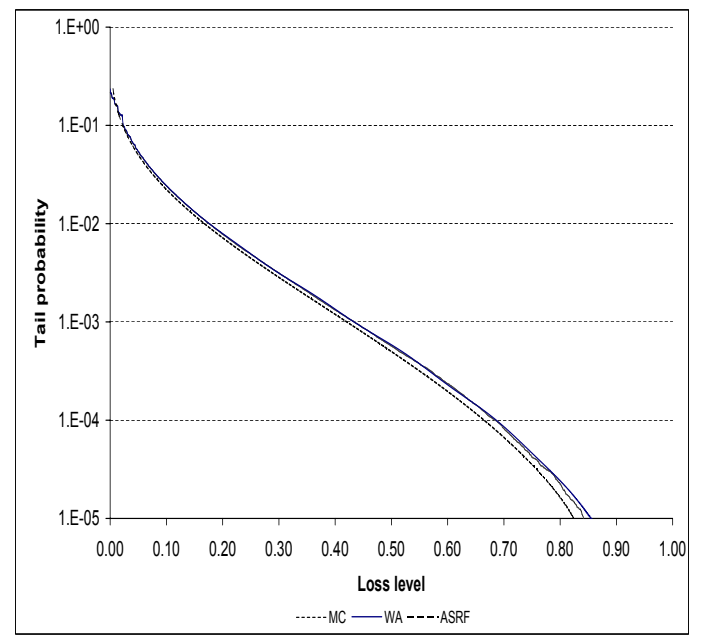

FiguRE 5. Tail probability approximation for portfolio 3 using WA with a 64-nodes GH formula and $\epsilon=10^{-1}$.

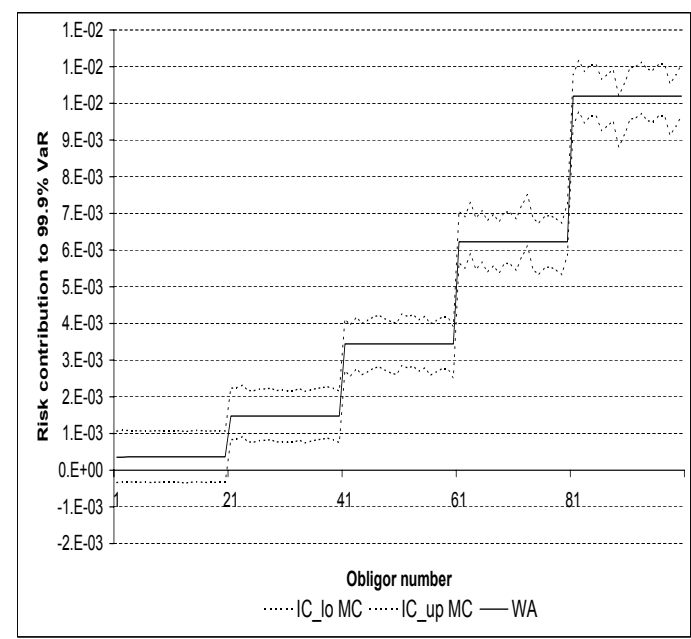

FiguRE 6. VaR contributions at $99.9 \%$ confidence level for portfolio 3 using the WA method and GH integration formulas with 64 nodes and $\bar{\epsilon}=10^{-4}$.

Finally we present the results for portfolio 4. The computation of the $99.99 \%$ ES and 99.99\% ESC have been carried out by means of the plain WA method with a 20-nodes GH formula, since this portfolio is extremely small and the computational effort is tinny to consider other improvements. The results are presented in tables 10 and 11. With this example we just want to remark that the WA method is very versatile and it can also deal with very small portfolios.

\section{Conclusions}

This paper extends a previous work undertaken in [Mas10]. It is based on a Haar wavelet approximation to the cumulative distribution of the loss function with the computation of the 

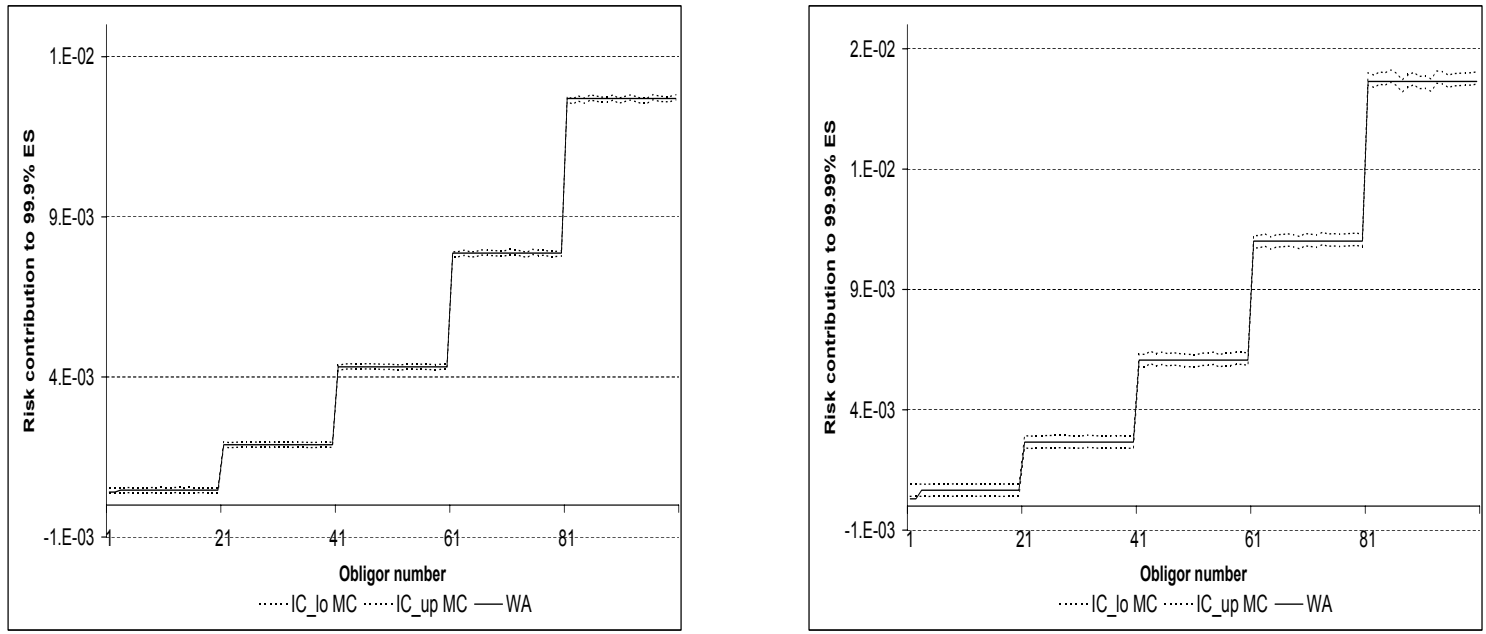

FiguRE 7. Expected Shortfall contributions at 99.9\% (left) and 99.99\% (right) confidence levels for portfolio 3 using the WA method and GH integration formulas with 64 nodes and $\bar{\epsilon}=10^{-4}$.

\begin{tabular}{ll}
\hline \hline Method & $\alpha=0.9999$ \\
\hline \hline $\mathrm{ES}_{\alpha}^{M}$ & 0.6833 \\
$\operatorname{ES}_{\alpha}^{W(10)}(20)$ & $0.6814(-0.29 \%)$
\end{tabular}

TABLE 10. ES at $99.99 \%$ confidence level for portfolio 4. Relative error to Monte Carlo is shown in parenthesis.

ES as an alternative coherent risk measure to VaR. Moreover, a detailed procedure for the calculation of the risk contributions to the VaR and the ES in a credit portfolio is provided. The risk contributions are known to be very computationally intensive to be estimated by means of MC because they are the expected value of the individual loss conditioned on a rare event. Therefore, analytical or fast numerical methods are welcome to overcome this problem. The model framework is the well known Vasicek one-factor model, a one-period default model, which is the basis of the Basel II Accord.

There are technical points taken into account that contribute to a considerable improvement of the WA method. We avoid the evaluation of the MFG in all the nodes of the Gauss-Hermite formulas by means of using its asymptotic behavior. Proceeding this way the speed of the WA method increases while accuracy is even improved with the choice of the parameter $r$. These improvements are also applied to the computation of risk contributions to VaR and ES, although the impact in the speed of the algorithm is much more relevant for risk measures than for risk contributions.

This new methodology has been tested in a wide sized variety of portfolios, all them with exposure concentration, where the Asymptotic Single Risk Factor Model fails due to the name concentration. The results presented show that the Wavelet Approximation method is highly competitive in terms of robustness, speed and accuracy being a very suitable method to measure and manage the risks that arise in credit portfolios of financial companies. 


\begin{tabular}{llll}
\hline \hline Obligor & $\mathrm{ESC}_{0.9999, n}^{W(20)}$ & $\mathrm{ESC}_{0.9999, n}^{M}$ & Relative error \\
\hline \hline 1 & 0.340472 & 0.338706 & $0.52 \%$ \\
2 & 0.128672 & 0.128885 & $-0.17 \%$ \\
3 & 0.059568 & 0.059426 & $0.24 \%$ \\
4 & 0.041619 & 0.042070 & $-1.07 \%$ \\
5 & 0.028044 & 0.028805 & $-2.64 \%$ \\
6 & 0.023233 & 0.022825 & $1.79 \%$ \\
7 & 0.019335 & 0.019348 & $-0.06 \%$ \\
8 & 0.016606 & 0.015935 & $4.21 \%$ \\
9 & 0.014527 & 0.014614 & $-0.60 \%$ \\
10 & 0.012971 & 0.012227 & $6.08 \%$ \\
\hline \hline$\sum_{n=1}^{N} \mathrm{ESC}_{0.9999, n}^{W(10)}(20)$ & 0.6850 & 0.6828 & $0.32 \%$ \\
\hline
\end{tabular}

TABLE 11. ES contributions at $99.99 \%$ confidence level for portfolio 4 with the WA method.

\section{REFERENCES}

[Art99] P. Artzner, F. Delbaen, J.M. Eber, D. Heath (1999). Coherent measures of risk. Mathematical Finance 9 (3): 203-228.

[Gla05] P. Glasserman (2005). Measuring marginal risk contributions in credit portfolios. Journal of Computational Finance. V. 9, N. 2, 1-41.

[Hua07a] X. Huang, C. W. Oosterlee and J. A. M. van der Weide (2007). Higher-order saddlepoint approximations in the Vasicek portfolio credit loss model. Journal of Computational Finance V. 11, N. 1, 93-113.

[Hua07b] X. Huang, C. W. Oosterlee and M. A. M. Mesters (2007). Computation of VaR and VaR contribution in the Vasicek portfolio credit loss model. Journal of Credit Risk V. 3, N. 3, 75-96.

[Lut09] E. Lütkebohmert (2009). Concentration risk in credit portfolios. Springer.

[Mar01a] R. Martin, K. Thompson and C. Browne (2001). Taking to the saddle. RISK (June), 91-94.

[Mar01b] R. Martin, K. Thompson and C. Browne (2001). VaR: who contributes and how much?. RISK (August), 99-102.

[Mas10] J. J. Masdemont and L. Ortiz-Gracia (2010). Haar wavelets-based approach for quantifying credit portfolio losses. Available at www.crm.cat.

[Tak08] Y. Takano and J. Hashiba (2008). A novel methodology for credit portfolio analysis: numerical approximation approach. Available at www.defaultrisk.com.

[Tas00] D. Tasche (2000). Risk contributions and performance measurement. Working paper, Munich University of Technology. 
Luis Ortiz-Gracia

Centre de Recerca Matemàtica

Campus de Bellaterra, Edifici C

08193 Bellaterra

(BARCElona), Spain

E-mail address: lortiz@crm.cat

Josep J. MASDEMONT

Departament de Matemàtica Aplicada I

Universitat Politècnica de Catalunya

Diagonal 647

08028 BarCELONA, Spain

E-mail address: josep@barquins.upc.edu 